Supporting Information

Electrooxidative Glycosylation thorough C-S Bond Cleavage of 1-Aryl-2,3-dideoxyglycosides. Synthesis of 2',3'-Dideoxynucleosides

Koichi Mitsudo, Takashi Kawaguchi, Seiji Miayahara, Wataru Matsuda, Manabu Kuroboshi, and Hideo Tanaka*

General. ${ }^{1} \mathrm{H}$ and ${ }^{13} \mathrm{C}$ NMR spectra were recorded on Varian GEMINI $200\left({ }^{1} \mathrm{H} 200 \mathrm{MHz},{ }^{13} \mathrm{C} 50 \mathrm{MHz}\right)$, MERCURY $300\left({ }^{1} \mathrm{H} 300 \mathrm{MHz},{ }^{13} \mathrm{C} 75 \mathrm{MHz}\right)$ and VXL $500\left({ }^{1} \mathrm{H} 500 \mathrm{MHz},{ }^{13} \mathrm{C} 125 \mathrm{MHz}\right)$ spectrometers in $\mathrm{CDCl}_{3}$ using TMS or residual chloroform as internal standard. IR spectra were recorded on a JASCO FT/IR-4100 spectrophotometer. Dichloromethane $\left(\mathrm{CH}_{2} \mathrm{Cl}_{2}\right)$ was pretreated with $\mathrm{P}_{4} \mathrm{O}_{10}$ and distilled over $\mathrm{CaH}_{2}$. Unless otherwise noted, all reactions were performed under argon.

General procedure for Preparation of 1-Arylthio-Substituted 2,3-Dideoxyglycoside. In the presence of montmorillonite $\mathrm{K}-10 \quad(0.345 \mathrm{~g})$, to a solution of 5-[(tert-butyldimethylsilanyloxy)methyl]tetrahydrofuran-2-ol $(89 \mathrm{mg}, 14 \mathrm{mmol})$ in $\mathrm{CH}_{2} \mathrm{Cl}_{2}(10 \mathrm{~mL})$, was added dropwise 3,5-di-tert-butyl-4-methoxybenzenethiol (124 mg, $0.49 \mathrm{mmol})$ at room temperature. After being stirred at room temperature for $4 \mathrm{~h}$, the reaction mixture was filtered and concentrated under reduced pressure. The residue was purified by chromatography on silica gel (hexane $/ \mathrm{CH}_{2} \mathrm{Cl}_{2}=2: 1$ ) to afford 1-(3',5'-di-tert-butyl-4'-methoxyphenylsulfanyl)-5-O-(tert-butyldimethylsilyl)-2,3-dideoxyglyceropentofuranose (1a, $127 \mathrm{mg}, 0.27 \mathrm{mmol}, 72 \%)$ as a colorless liquid.

1-(3',5'-Di-tert-butyl-4'-methoxyphenylsulfanyl)-5- $O$-(tert-butyldimethylsilyl)-2,3-dideoxy- $\alpha$-Dglycero-pentofuranose $(\mathbf{1 a} \alpha) .{ }^{1} \mathrm{H} \mathrm{NMR}\left(500 \mathrm{MHz}, \mathrm{CDCl}_{3}\right) \delta 0.05(\mathrm{~s}, 6 \mathrm{H}), 0.88(\mathrm{~s}, 9 \mathrm{H}), 1.41(\mathrm{~s}, 18 \mathrm{H})$, 1.74-1.80 (m, 1H), 1.94-2.05 (m, 2H), 2.34-2.40 (m, 1H), $3.67(\mathrm{~s}, 3 \mathrm{H}), 3.68(\mathrm{~d}, J=5.5 \mathrm{~Hz}, 2 \mathrm{H})$, $4.26-4.31(\mathrm{~m}, 1 \mathrm{H}), 5.58(\mathrm{dd}, J=7.0,3.5 \mathrm{~Hz}, 1 \mathrm{H}), 7.40(\mathrm{~s}, 2 \mathrm{H}) ;{ }^{13} \mathrm{C} \mathrm{NMR}\left(125 \mathrm{MHz}, \mathrm{CDCl}_{3}\right) \delta-5.3,18.3$, 25.9, 26.7, 32.0, 32.7, 35.7, 64.2, 65.0, 79.1, 88.1, 128.4, 130.6, 144.0, 159.0; IR (neat) 3006, 2956, 2860, $1574,1463,1408,1254,1222,1116,1011,839 \mathrm{~cm}^{-1}$.

1-(3',5'-Di-tert-butyl-4'-methoxyphenylsulfanyl)-5- $O$-(tert-butyldimethylsilyl)-2,3-dideoxy- $\beta$-Dglycero-pentofuranose $(\mathbf{1 a} \beta) .{ }^{1} \mathrm{H} \mathrm{NMR}\left(500 \mathrm{MHz}, \mathrm{CDCl}_{3}\right) \delta 0.06(\mathrm{~s}, 6 \mathrm{H}), 0.90(\mathrm{~s}, 9 \mathrm{H}), 1.41(\mathrm{~s}, 18 \mathrm{H})$, $1.85-1.93(\mathrm{~m}, 1 \mathrm{H}), 2.00-2.11(\mathrm{~m}, 2 \mathrm{H}), 2.26-2.34(\mathrm{~m}, 1 \mathrm{H}), 3.63(\mathrm{dd}, J=10.5,6.0 \mathrm{~Hz}, 1 \mathrm{H}), 3.67(\mathrm{~s}, 3 \mathrm{H})$, $3.79(\mathrm{dd}, J=10.5,6.0 \mathrm{~Hz}, 1 \mathrm{H}), 4.10-4.15(\mathrm{~m}, 1 \mathrm{H}), 5.45(\mathrm{dd}, J=7.0,4.5 \mathrm{~Hz}, 1 \mathrm{H}), 7.39(\mathrm{~s}, 2 \mathrm{H}){ }^{13} \mathrm{C} \mathrm{NMR}$ $\left(125 \mathrm{MHz}, \mathrm{CDCl}_{3}\right) \delta-5.3,18.3,25.9,27.9,32.0,32.8,35.8,64.2,66.3,81.7,88.0,128.0,131.0,144.1$, 159.2; IR (neat) 3011, 2955, 2858, 1572, 1471, 1408, 1256, 1223, 1077, 1010, $838 \mathrm{~cm}^{-1}$. Anal. Calcd for $\mathrm{C}_{26} \mathrm{H}_{46} \mathrm{O}_{3} \mathrm{SSi}$ : C, 66.90; H, 9.93. Found: C, 66.97; H, 10.18 . 
In a similar manner, glycosyl donors $\mathbf{1 b}$ and $\mathbf{1 c}$ were prepared. The results are summarized in Table 1.

Table 1. Preparation of Glycosyl Donors 1a-1c.

entry the (h) $\quad$ thioglycoside $\mathbf{1}^{a} \quad$ yield $(\%)^{b} \quad \alpha / \beta^{c}$

${ }^{a} \mathrm{R}=$ TBDMS. ${ }^{b}$ Isolated yield. ${ }^{c}$ Determined by ${ }^{1} \mathrm{H}$ NMR.

1-(9'-Anthracenethionyl)-5-O-(tert-butyldimethylsilyl)-2,3-dideoxy- $\alpha$-D-glycero-pentofuranose (1bo). A yellow liquid; ${ }^{1} \mathrm{H}$ NMR $\left(500 \mathrm{MHz}, \mathrm{CDCl}_{3}\right) \delta 0.07(\mathrm{~s}, 6 \mathrm{H}), 0.82(\mathrm{~s}, 9 \mathrm{H}), 1.72-1.77(\mathrm{~m}, 1 \mathrm{H})$, 2.10-2.19 (m, 2H), 2.27-2.33 (m, 1H), $3.48(\mathrm{dd}, J=11.0,5.0 \mathrm{~Hz}, 1 \mathrm{H}), 3.53(\mathrm{dd}, J=11.0,5.0 \mathrm{~Hz}, 1 \mathrm{H})$, 4.30-4.34 (m, 1H), 5.60 (dd, $J=6.0,2.0 \mathrm{~Hz}, 1 \mathrm{H}), 7.49$ (t, $J=6.5 \mathrm{~Hz}, 2 \mathrm{H}), 7.57(\mathrm{t}, J=6.5 \mathrm{~Hz}, 2 \mathrm{H}), 8.00$ $(\mathrm{d}, J=9.0 \mathrm{~Hz}, 2 \mathrm{H}), 8.49(\mathrm{~s}, 1 \mathrm{H}), 8.96(\mathrm{~d}, J=9.0 \mathrm{~Hz}, 2 \mathrm{H}) ;{ }^{13} \mathrm{C} \mathrm{NMR}\left(125 \mathrm{MHz}, \mathrm{CDCl}_{3}\right) \delta-5.4,18.2,25.8$, 26.8, 33.0, 65.0, 79.7, 89.6, 125.2, 126.4, 127.3, 128.0, 128.8, 129.1, 131.8, 135.1; IR (neat) 3076, 3049, $2955,2926,1517,1461,1361,1309,1257,1073,838 \mathrm{~cm}^{-1}$.

\section{1-(9'-Anthracenethionyl)-5-O-(tert-butyldimethylsilyl)-2,3-dideoxy- $\beta$-D-glycero-pentofuranose} (1b $\beta)$. Yellow solids; ${ }^{1} \mathrm{H}$ NMR $\left(500 \mathrm{MHz}, \mathrm{CDCl}_{3}\right) \delta 0.06(\mathrm{~s}, 6 \mathrm{H}), 0.92(\mathrm{~s}, 9 \mathrm{H}), 1.95-1.98(\mathrm{~m}, 1 \mathrm{H})$, 2.06-2.12 (m, 2H), 2.15-2.22 (m, 1H), 3.52 (dd, $J=10.0,6.0 \mathrm{~Hz}, 1 \mathrm{H}), 3.67$ (dd, $J=10.0,6.0 \mathrm{~Hz}, 1 \mathrm{H})$, 3.99-4.04 (m, 1H), 5.45 (dd, $J=6.0,2.5 \mathrm{~Hz}, 1 \mathrm{H}), 7.49$ (t, $J=7.0 \mathrm{~Hz}, 2 \mathrm{H}), 7.58(\mathrm{t}, J=7.0 \mathrm{~Hz}, 2 \mathrm{H}), 8.01$ $(\mathrm{d}, J=8.5 \mathrm{~Hz}, 2 \mathrm{H}), 8.49(\mathrm{~s}, 1 \mathrm{H}), 8.98(\mathrm{~d}, J=8.5 \mathrm{~Hz}, 2 \mathrm{H}) ;{ }^{13} \mathrm{C} \mathrm{NMR}\left(125 \mathrm{MHz}, \mathrm{CDCl}_{3}\right) \delta-5.3,18.4,26.0$, 27.6, 33.4, 65.9, 81.8, 89.4, 125.3, 126.4, 127.5, 127.8, 128.8, 129.1, 131.8, 135.0; IR (KBr) 3048, 2929, 2855, 1620, 1463, 1253, 1136, 1004, $836 \mathrm{~cm}^{-1}$; Anal. Calcd for $\mathrm{C}_{25} \mathrm{H}_{32} \mathrm{O}_{2} \mathrm{SSi}$ : C, 70.71; H, 7.60. Found: C, 70.87; H, 7.67 .

\section{1-[4'-(N,N-Diphenylamino)phenylsulfanyl]-5-O-(tert-butyldimethylsilyl)-2,3-dideoxy- $\alpha$-D-}


glycero-pentofuranose (1c $\alpha)$. A light yellow liquid; ${ }^{1} \mathrm{H}$ NMR $\left(500 \mathrm{MHz}, \mathrm{CDCl}_{3}\right) \delta 0.05(\mathrm{~s}, 6 \mathrm{H}), 0.87(\mathrm{~s}$, 9H), 1.72-1.79 (m, 1H), 1.94-2.08 (m, 2H), 2.34-2.41 (m, 1H), $3.68(\mathrm{~d}, J=4.0 \mathrm{~Hz}, 2 \mathrm{H}), 4.28-4.32(\mathrm{~m}$, $1 \mathrm{H}), 5.59(\mathrm{dd}, J=7.0,4.5 \mathrm{~Hz}, 1 \mathrm{H}), 6.97-7.03(\mathrm{~m}, 4 \mathrm{H}), 7.08(\mathrm{~d}, J=7.5 \mathrm{~Hz}, 4 \mathrm{H}), 7.24(\mathrm{t}, J=7.5 \mathrm{~Hz}, 2 \mathrm{H})$, $7.37(\mathrm{~d}, J=8.5 \mathrm{~Hz}, 2 \mathrm{H}) ;{ }^{13} \mathrm{C} \mathrm{NMR}\left(125 \mathrm{MHz}, \mathrm{CDCl}_{3}\right) \delta-5.3,18.3,25.9,26.8,32.6,65.0,79.2,88.2$, 122.9, 123.8, 124.4, 128.0, 129.2, 133.2, 147.1, 147.5; IR (neat) 3063, 3036, 2954, 2929, 1586, 1493, $1315,1277,1070,998,838 \mathrm{~cm}^{-1}$.

1-[4'-( $N, N$-Diphenylamino)phenylsulfanyl]-5- $O$-(tert-butyldimethylsilyl)-2,3-dideoxy- $\beta$-Dglycero-pentofuranose (1c $\beta)$. A light yellow liquid; ${ }^{1} \mathrm{H} \mathrm{NMR}\left(500 \mathrm{MHz}, \mathrm{CDCl}_{3}\right) \delta 0.06(\mathrm{~s}, 6 \mathrm{H}), 0.89(\mathrm{~s}$, 9H), 1.85-1.93 (m, 1H), 2.00-2.10 (m, 2H), 2.28-2.35 (m, 1H), 3.63 (dd, J=10.5, 6.5 Hz, 1H), 3.78 (dd, $J=10.5,6.5 \mathrm{~Hz}, 1 \mathrm{H}), 4.13-4.18(\mathrm{~m}, 1 \mathrm{H}), 5.47(\mathrm{dd}, J=7.0,2.5 \mathrm{~Hz}, 1 \mathrm{H}), 6.97-7.03(\mathrm{~m}, 4 \mathrm{H}), 7.08(\mathrm{~d}, J=$ $7.0 \mathrm{~Hz}, 4 \mathrm{H}), 7.25(\mathrm{t}, J=8.5 \mathrm{~Hz}, 2 \mathrm{H}), 7.37(\mathrm{~d}, J=8.5 \mathrm{~Hz}, 2 \mathrm{H}) ;{ }^{13} \mathrm{C} \mathrm{NMR}\left(125 \mathrm{MHz}, \mathrm{CDCl}_{3}\right) \delta-5.3,18.3$, 25.9, 27.8, 32.7, 66.2, 81.8, 87.9, 123.0, 123.6, 124.4, 127.5, 129.2, 133.4, 147.1, 147.4; IR (neat) 3062,

3036, 2953, 2928, 1586, 1490, 1314, 1279, 1076, 1051, $837 \mathrm{~cm}^{-1}$; Anal. Calcd for $\mathrm{C}_{29} \mathrm{H}_{37} \mathrm{NO}_{2} \mathrm{SSi}: \mathrm{C}$, 70.83; H, 7.58; N, 2.85. Found: C, 70.89; H, 7.77; N, 2.74.

\section{Typical Procedure for $\left[\mathrm{X}^{-}\right] /\left[\mathrm{X}^{+}\right]$-Mediated Electrooxidative Glycosylation of Nucleobases with} 1a-1c. In a beaker-type undivided cell, equipped two platinum electrodes $\left(1.0 \times 1.0 \mathrm{~cm}^{2}\right)$, were placed thioglycoside $1 \mathrm{a} \alpha$ (47 $\mathrm{mg}, 0.10 \mathrm{mmol}$ ), tetrabutylammonium bromide (41 mg, $0.13 \mathrm{mmol}$ ), TMS-uracil $2(103 \mathrm{mg}, 0.40 \mathrm{mmol})$, and $\mathrm{CH}_{2} \mathrm{Cl}_{2}(6.0 \mathrm{~mL})$. Electrolysis was carried out at $-78{ }^{\circ} \mathrm{C}$ under constant current conditions (10 mA, $32 \mathrm{~min}, 2 \mathrm{~F} / \mathrm{mol})$. The resultant was allowed to warm to room temperature, and concentrated under reduced pressure. The residue was dissolved in EtOAc, and washed with brine and dried over $\mathrm{Na}_{2} \mathrm{SO}_{4}$. After removal of the solvent in vacuo, the residue was purified by column chromatography on silica gel (hexane/EtOAc 1:1) to afford nucleoside 3 (26 mg, 0.79 mmol, 79\%, $\beta / \alpha=$ 3.6) as a colorless liquid.

\section{Typical Procedure for $\left[\mathrm{X}^{-}\right] /\left[\mathrm{X}^{+}\right]$-Mediated Step-wise Electrooxidative Glycosylation of} Nucleobases with 1a-1c. In a beaker-type undivided cell, equipped two platinum electrodes $(1.0 \times 1.0$ $\mathrm{cm}^{2}$ ), were placed 2,3-dideoxyglycoside $1 \mathbf{a} \alpha(44 \mathrm{mg}, 0.10 \mathrm{mmol})$, tetrabutylammonium bromide (39 mg, $0.13 \mathrm{mmol})$ and $\mathrm{CH}_{2} \mathrm{Cl}_{2}(3.0 \mathrm{~mL})$. Electrolysis was carried out at $-78{ }^{\circ} \mathrm{C}$ under constant current conditions $(10 \mathrm{~mA}, 24 \mathrm{~min}, 1.5 \mathrm{~F} / \mathrm{mol})$. After the electrolysis, to the mixture was added dropwise a solution of TMS-uracil $(2,98 \mathrm{mg}, 0.38 \mathrm{mmol})$ in $\mathrm{CH}_{2} \mathrm{Cl}_{2}(1.0 \mathrm{~mL})$, and stirred for $60 \mathrm{~min}$ at $-78^{\circ} \mathrm{C}$. The resultant was allowed to warm to room temperature, and concentrated under reduced pressure. The residue was dissolved in EtOAc, and washed with brine and dried over $\mathrm{Na}_{2} \mathrm{SO}_{4}$. After removal of the solvent, the residue was purified by column chromatography on silica gel (hexane/EtOAc 1:1) to afford nucleoside 3 (25 mg, $0.77 \mathrm{mmol}, 81 \%, \beta / \alpha=5.1$ ) as a colorless liquid. 
$\mathbf{1}^{\prime}$-[5'-O-(tert-Butyldimethylsilyl)-2', $\mathbf{3}^{\prime}$-dideoxy- $\alpha$-D-glycero-pentofuranosyl]uracil $\quad(3 \alpha) . \quad$ A colorless liquid; ${ }^{1} \mathrm{H}$ NMR $\left(500 \mathrm{MHz}, \mathrm{CDCl}_{3}\right) \delta 0.08(\mathrm{~s}, 6 \mathrm{H}), 0.90(\mathrm{~s}, 9 \mathrm{H}), 1.93-2.10(\mathrm{~m}, 4 \mathrm{H}), 3.58-3.78$ $(\mathrm{m}, 2 \mathrm{H}), 4.39-4.48(\mathrm{~m}, 1 \mathrm{H}), 5.73(\mathrm{~d}, J=8.0 \mathrm{~Hz}, 1 \mathrm{H}), 6.02-6.05(\mathrm{~m}, 1 \mathrm{H}), 7.37(\mathrm{~d}, J=8.0 \mathrm{~Hz}, 1 \mathrm{H}) ; \mathrm{IR}$ (neat) $3136,2955,1683,1466,1389,1263,1085 \mathrm{~cm}^{-1}$.

$1^{\prime}$-[5'-O-(tert-Butyldimethylsilyl)-2', $\mathbf{3}^{\prime}$-dideoxy- $\beta$-D-glycero-pentofuranosyl]uracil (3ß). A colorless liquid; ${ }^{1} \mathrm{H}$ NMR $\left(500 \mathrm{MHz}, \mathrm{CDCl}_{3}\right) \delta 0.11(\mathrm{~s}, 6 \mathrm{H}), 0.93(\mathrm{~s}, 9 \mathrm{H}), 1.94-2.09(\mathrm{~m}, 3 \mathrm{H}), 2.34-2.43$ (m, 1H), $3.71(\mathrm{dd}, J=11.5,2.5 \mathrm{~Hz}, 1 \mathrm{H}), 4.07$ (dd, $J=11.5,2.51 \mathrm{H}), 4.15-4.18(\mathrm{~m}, 1 \mathrm{H}), 5.64(\mathrm{~d}, J=8.5$ $\mathrm{Hz}, 1 \mathrm{H}$ ), 6.07 (dd, $J=6.0,3.0 \mathrm{~Hz}, 1 \mathrm{H}$ ), 8.10 (d, $J=8.5 \mathrm{~Hz}, 1 \mathrm{H}$ ), 9.08 (brs, 1H); IR (neat) 3440, 2957, $2858,1683,1464,1393,1264,1185,1106,1002 \mathrm{~cm}^{-1}$.

$\mathbf{1}^{\prime}$-[5'-O-(tert-Butyldimethylsilyl)-2',3'-dideoxy-D-glycero-pentofuranosyl]cytosine (4). White solids; ${ }^{1} \mathrm{H}$ NMR (200 MHz, $\left.\mathrm{CDCl}_{3}\right) \delta 0.06$ and 0.09 (s, 6H), 0.89 and $0.91(\mathrm{~s}, 9 \mathrm{H}), 1.74-2.18(\mathrm{~m}, 3 \mathrm{H})$, 2.32-2.54 (m, $1 \mathrm{H}), 3.60-3.78$ and $4.02-4.10(\mathrm{~m}, 2 \mathrm{H}), 4.04-4.20$ and $4.31-4.43(\mathrm{~m}, 1 \mathrm{H}), 5.61(\mathrm{~d}, J=7.5$ $\mathrm{Hz})$ and $5.74(\mathrm{~d}, J=7.2 \mathrm{~Hz}, 1 \mathrm{H}), 6.07(\mathrm{dd}, J=6.6,4.0 \mathrm{~Hz}, 1 \mathrm{H}), 7.46(\mathrm{~d}, J=7.4 \mathrm{~Hz})$ and $8.17(\mathrm{~d}, J=7.5$ $\mathrm{Hz}, 1 \mathrm{H})$; IR (KBr) 3352, 3111, 2929, 2857, 1667, 1620, 1484, 1280, 1092, $838 \mathrm{~cm}^{-1}$.

$1^{\prime}$-[5'-O-(tert-Butyldimethylsilyl)-2',3'-dideoxy-D-glycero-pentofuranosyl]thymine (5). White solids; ${ }^{1} \mathrm{H}$ NMR $\left(200 \mathrm{MHz}, \mathrm{CDCl}_{3}\right) \delta 0.08$ and $0.11(\mathrm{~s}, 6 \mathrm{H}), 0.92$ and $0.95(\mathrm{~s}, 9 \mathrm{H}), 1.91-2.08(\mathrm{~m}, 3 \mathrm{H})$, 1.92 and $1.94(\mathrm{~s}, 3 \mathrm{H}), 2.12-2.43(\mathrm{~m}, 1 \mathrm{H}), 3.64-3.75$ and 3.96-4.04 $(\mathrm{m}, 1 \mathrm{H}), 4.09-4.20$ and 4.38-4.47 (m, $1 \mathrm{H}), 6.09$ (dd, $J=6.2,4.6 \mathrm{~Hz}, 1 \mathrm{H}), 7.16$ and 7.59 (s, 1H), 8.98 (br, 2H); IR (KBr) 3466, 3175, 3057, 2928, $1704,1471,1263,1151 \mathrm{~cm}^{-1}$.

$\mathbf{1}^{\prime}$-[5'-O-(tert-Butyldimethylsilyl)-2' $\mathbf{3}^{\prime}$-dideoxy-D-glycero-pentofuranosyl]adenine (6). White solids; ${ }^{1} \mathrm{H}$ NMR (200 MHz, CDCl $) \delta 0.09$ and $0.10(\mathrm{~s}, 6 \mathrm{H}), 0.93$ and $0.94(\mathrm{~s}, 9 \mathrm{H}), 1.80-2.90(\mathrm{~m}, 4 \mathrm{H})$, $3.62-4.41(\mathrm{~m}, 3 \mathrm{H}), 4.48-4.72(\mathrm{~m}, 1 \mathrm{H}), 6.34(\mathrm{dd}, J=6.4,3.2 \mathrm{~Hz})$ and $6.55(\mathrm{dd}, J=6.4,2.0 \mathrm{~Hz}, 1 \mathrm{H}), 7.93$ and $8.35(\mathrm{~s}, 0.19 \mathrm{H}), 8.24$ and $8.84(\mathrm{~s}, 0.81 \mathrm{H}), 8.04$ and $8.05(\mathrm{~s}, 0.81 \mathrm{H}), 8.31$ and $8.34(\mathrm{~s}, 0.19 \mathrm{H})$; IR (KBr) $3117,2927,2856,1651,1409,1258,1081,838 \mathrm{~cm}^{-1}$. 
${ }^{1} \mathrm{H}$ NMR of $\mathbf{1 a} \alpha$

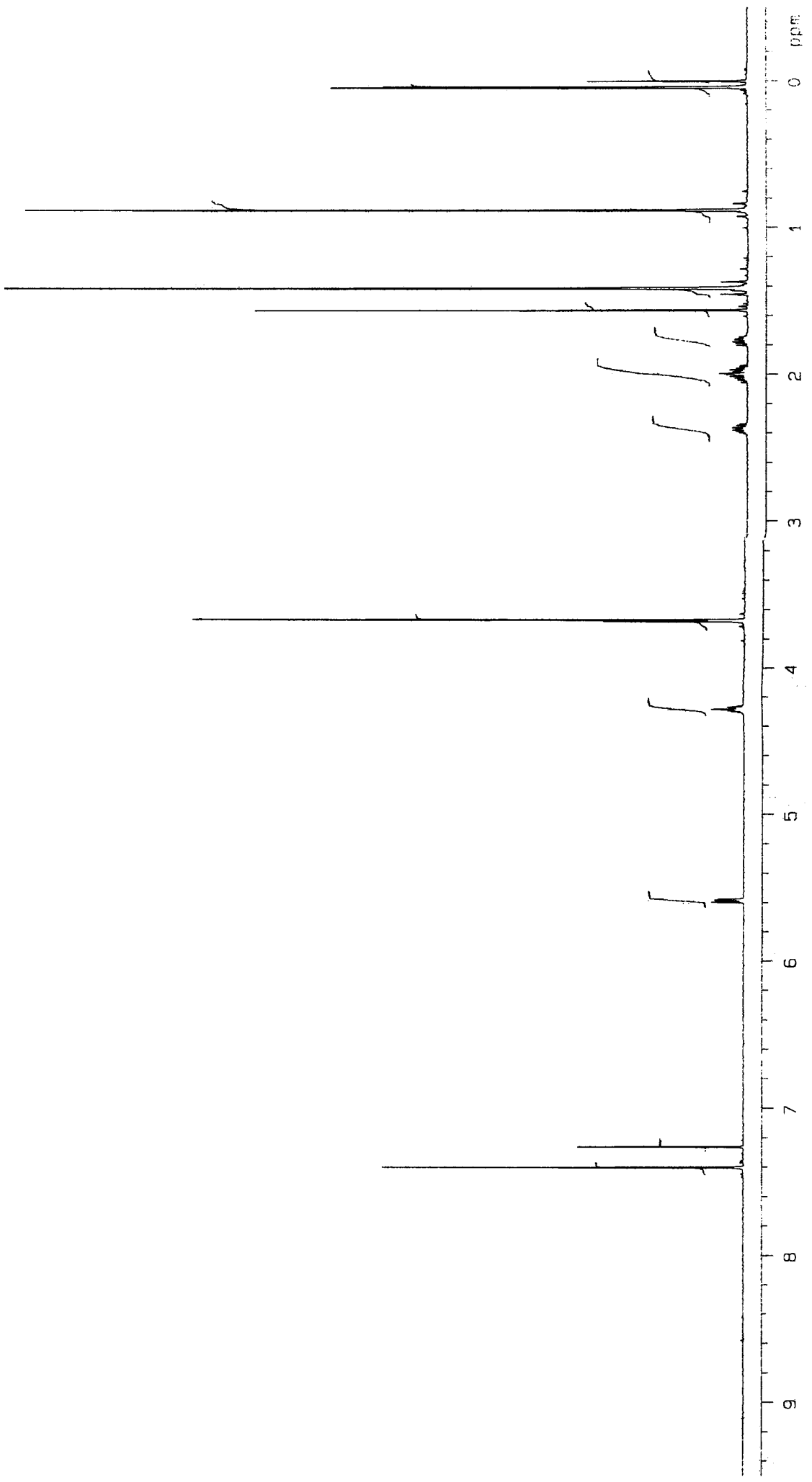




\section{${ }^{13} \mathrm{C}$ NMR of $\mathbf{1 a} \alpha$}

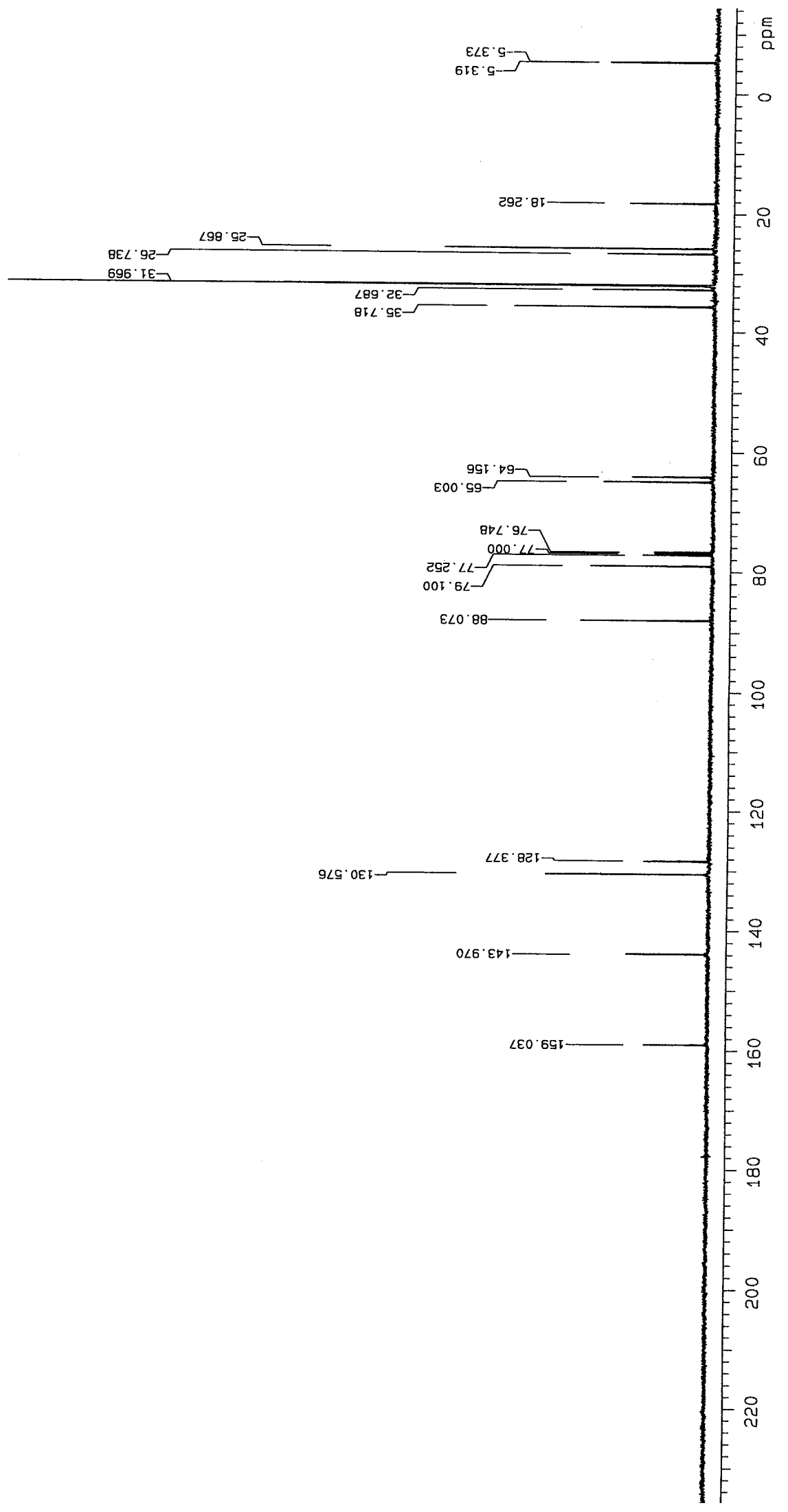



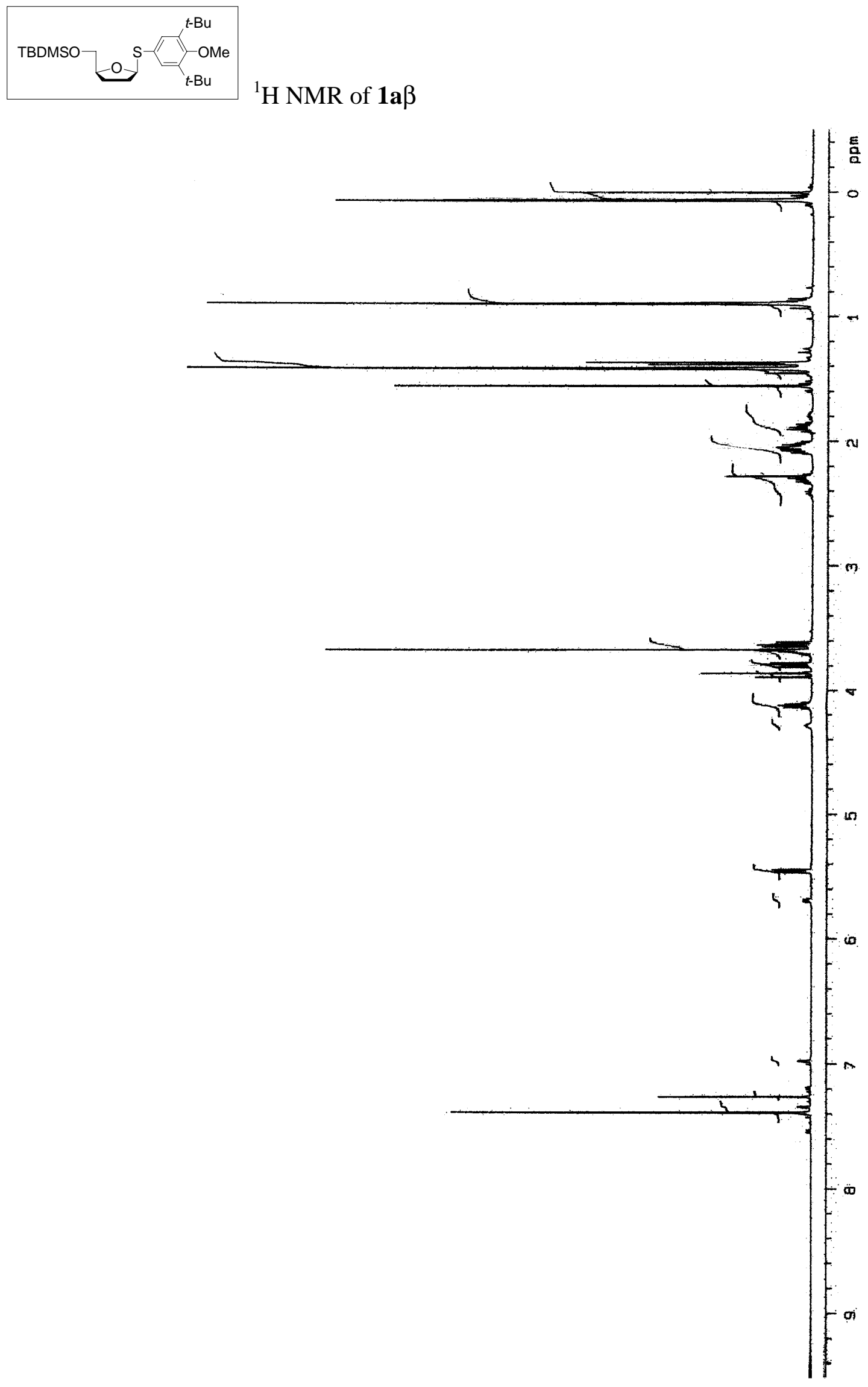
${ }^{13} \mathrm{C}$ NMR of $\mathbf{1 a} \beta$

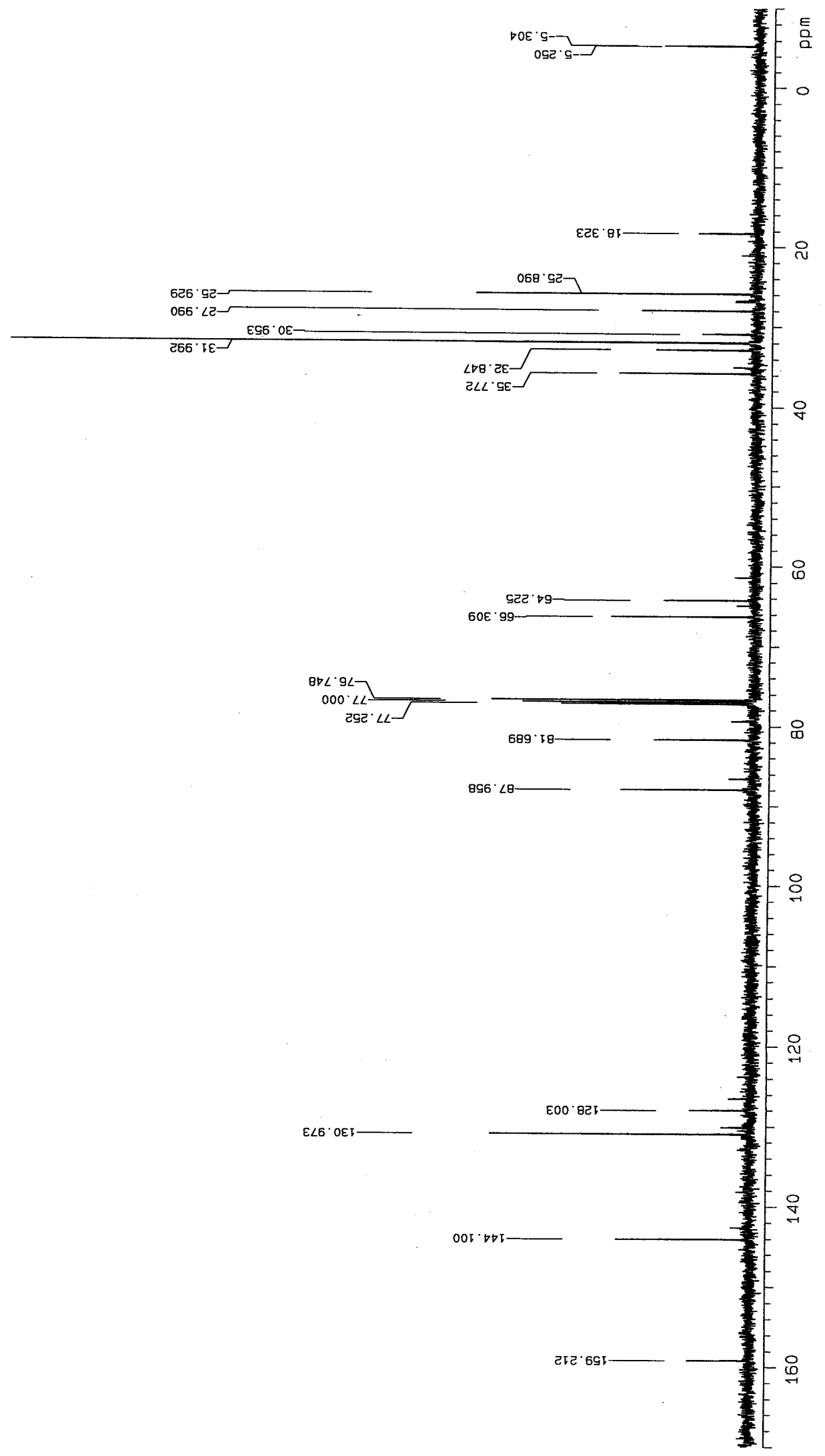

So 


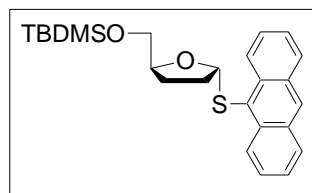

${ }^{1} \mathrm{H}$ NMR of $\mathbf{1 b} \alpha$

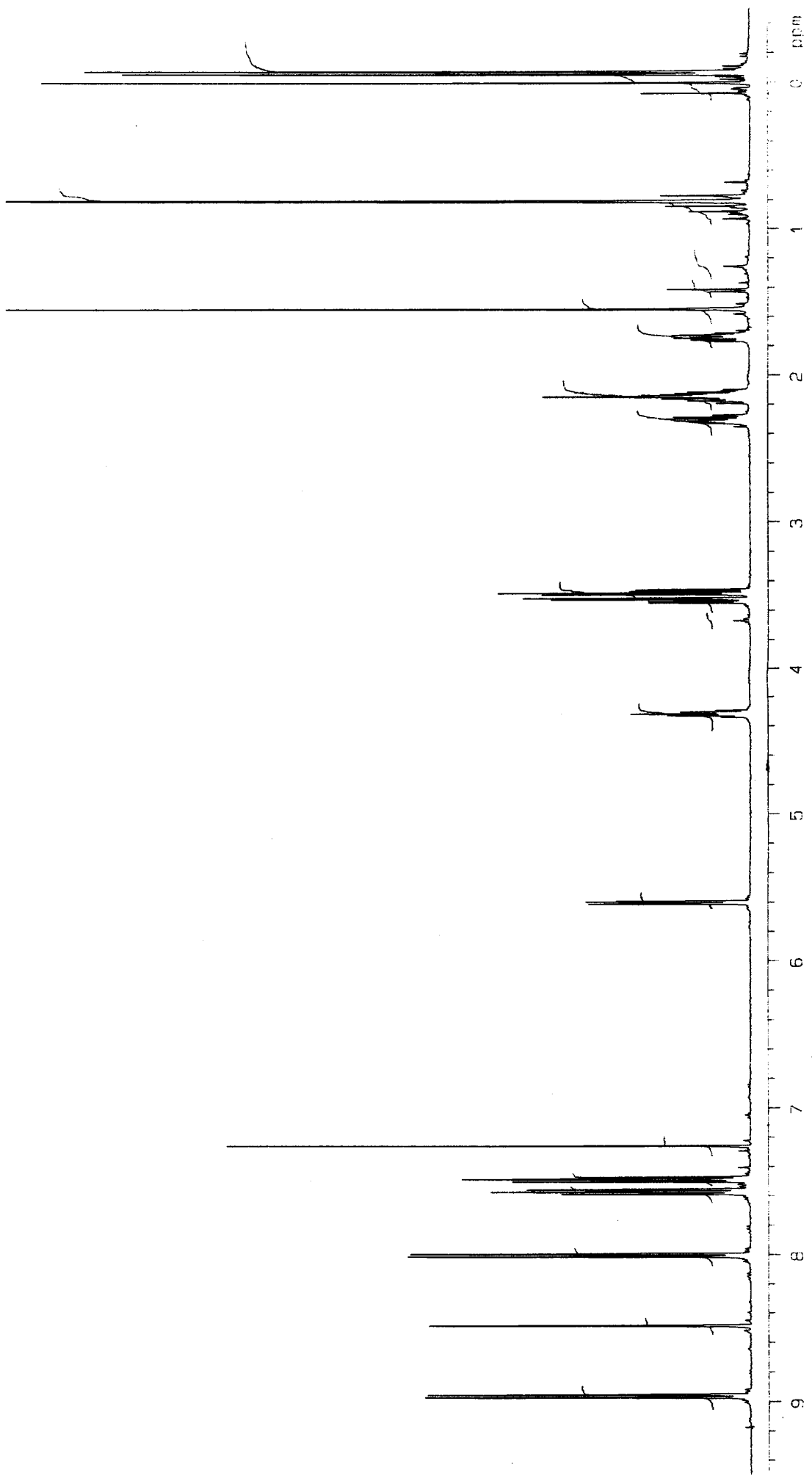




\section{${ }^{13} \mathrm{C}$ NMR of $\mathbf{1 b} \alpha$}

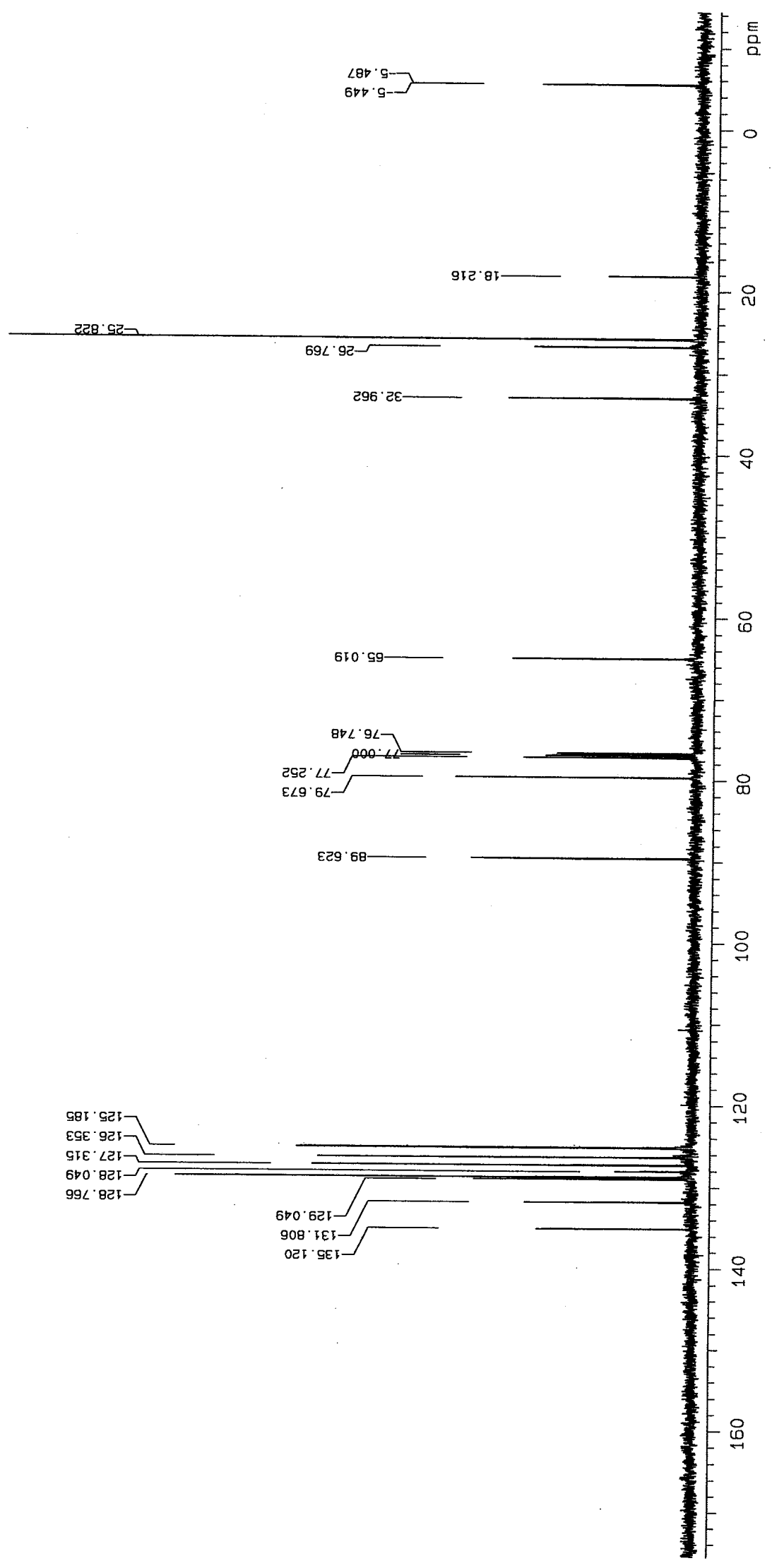




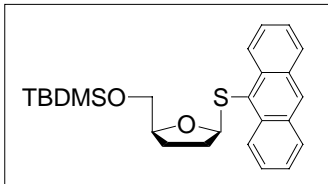

${ }^{1} \mathrm{H}$ NMR of $\mathbf{1 b} \beta$

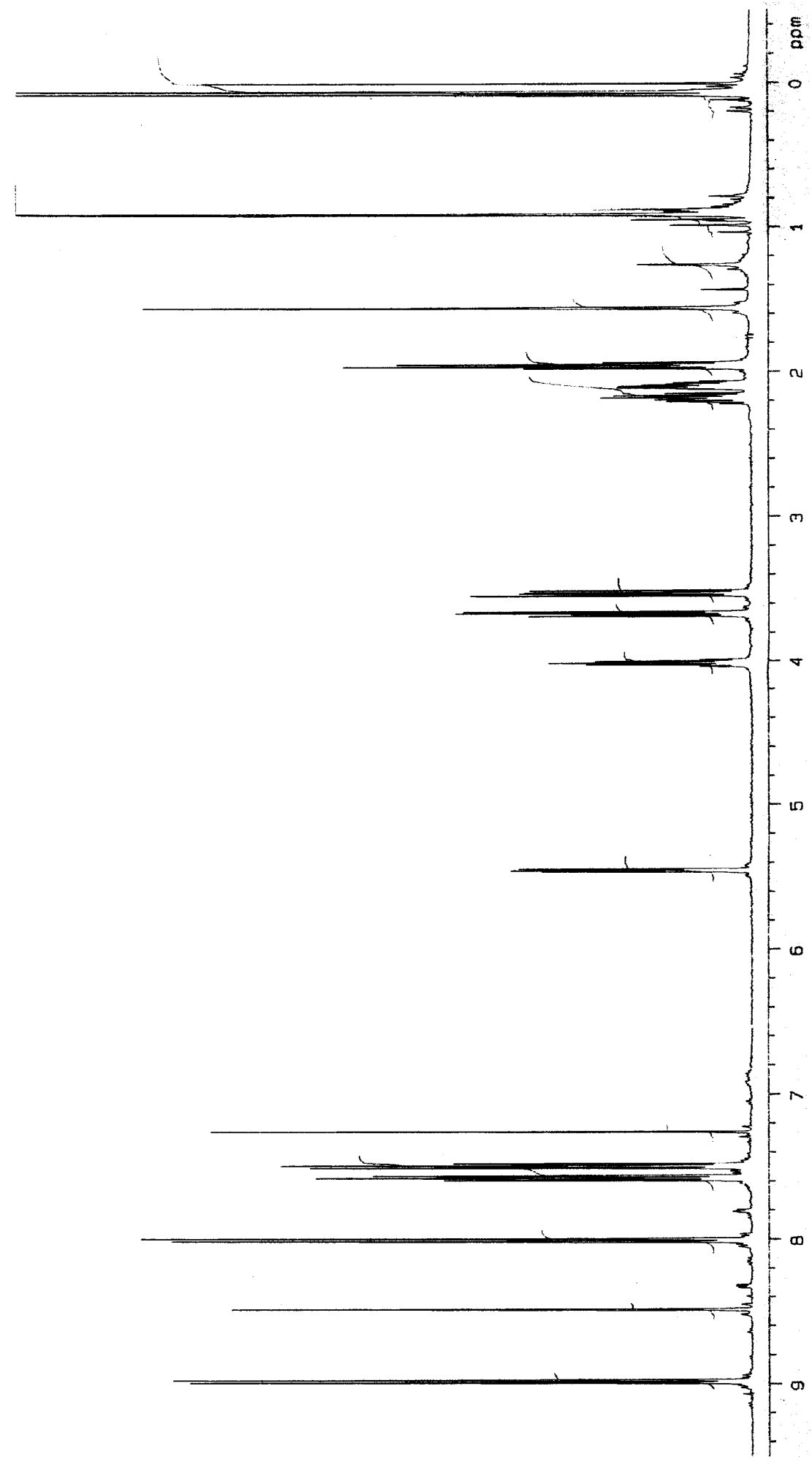


${ }^{13} \mathrm{C}$ NMR of $\mathbf{1 b} \beta$

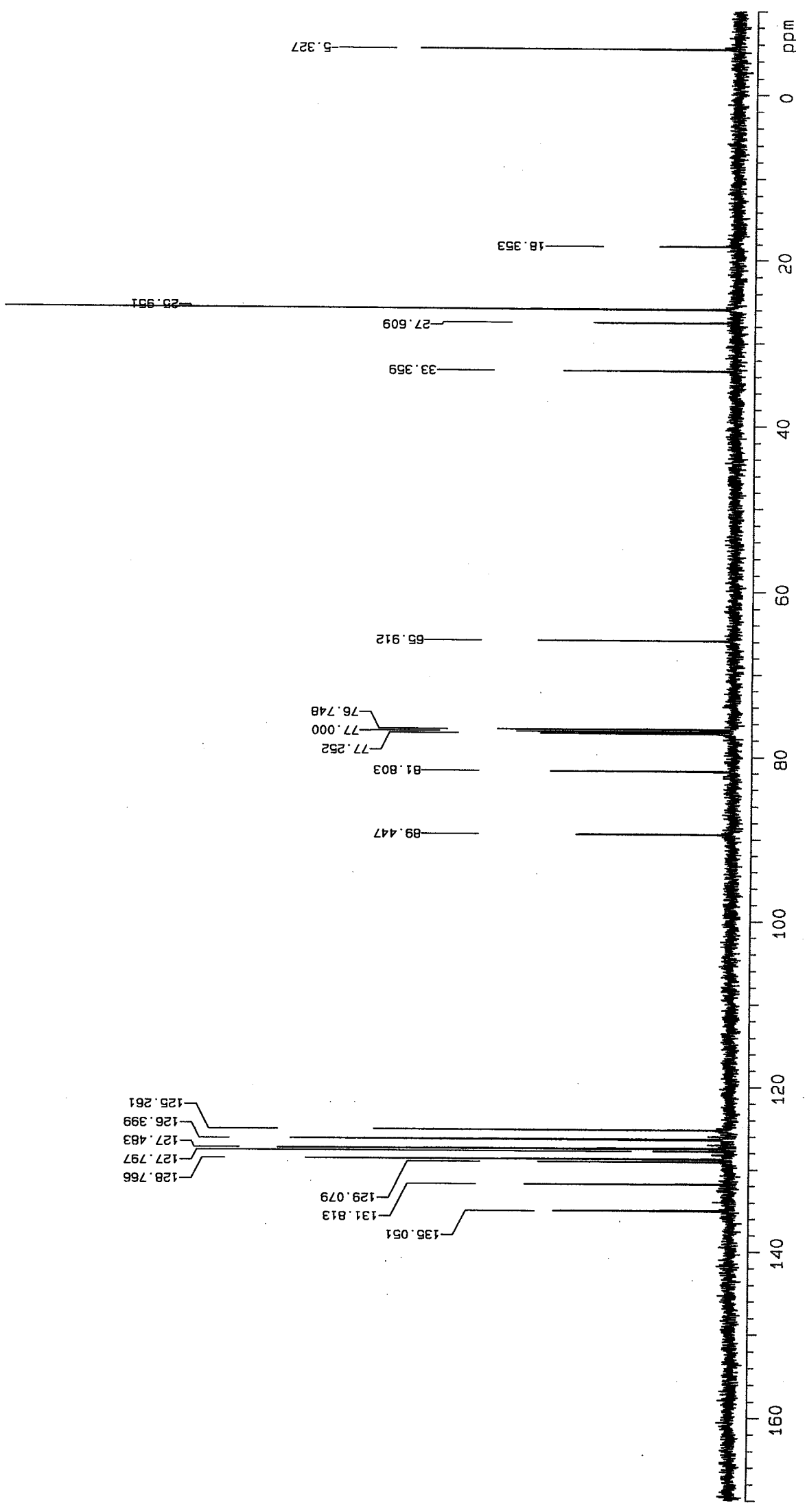


${ }^{1} \mathrm{H}$ NMR of $\mathbf{1 c} \alpha$

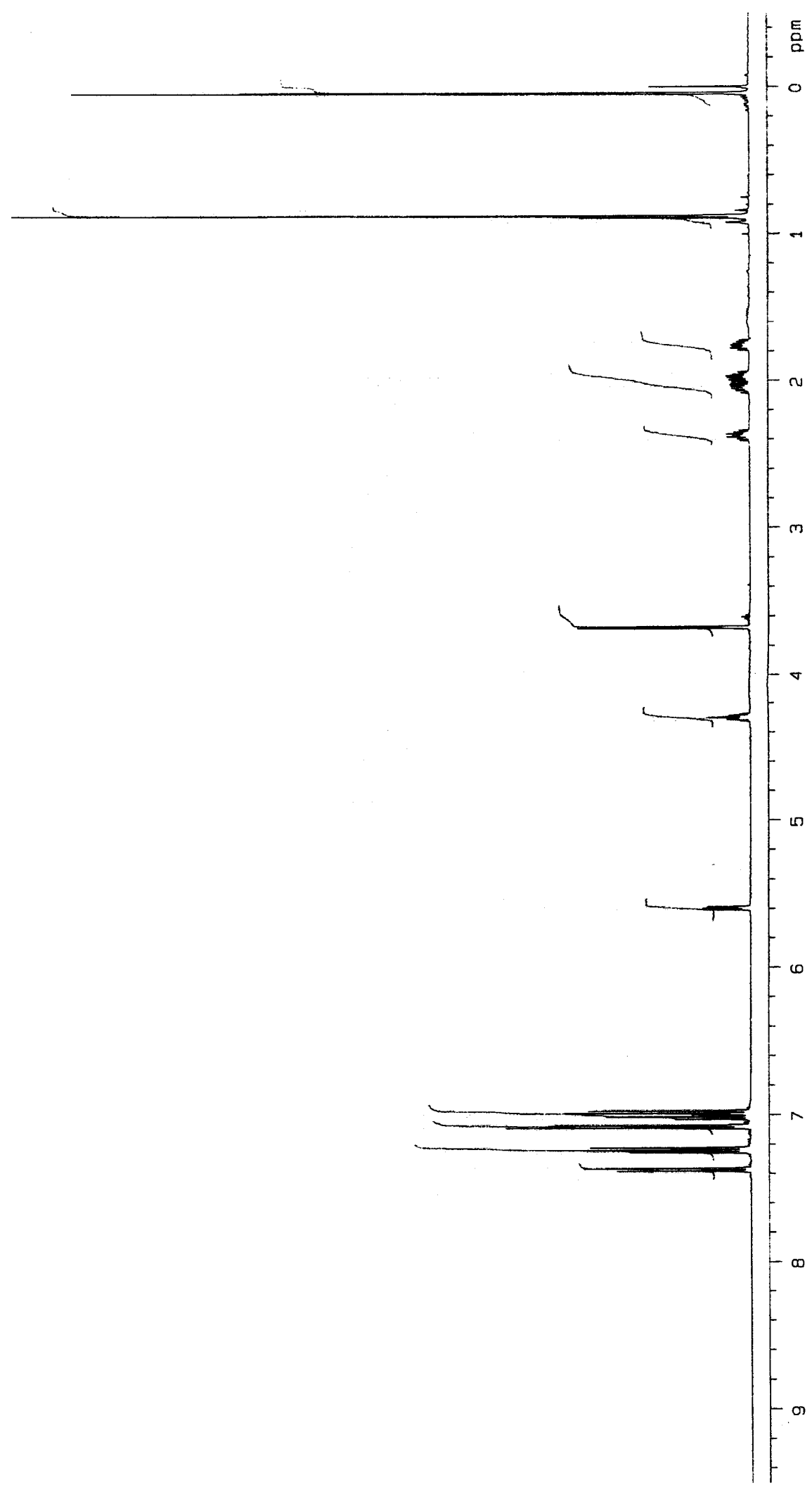


${ }^{13} \mathrm{C}$ NMR of $1 \mathrm{c} \alpha$

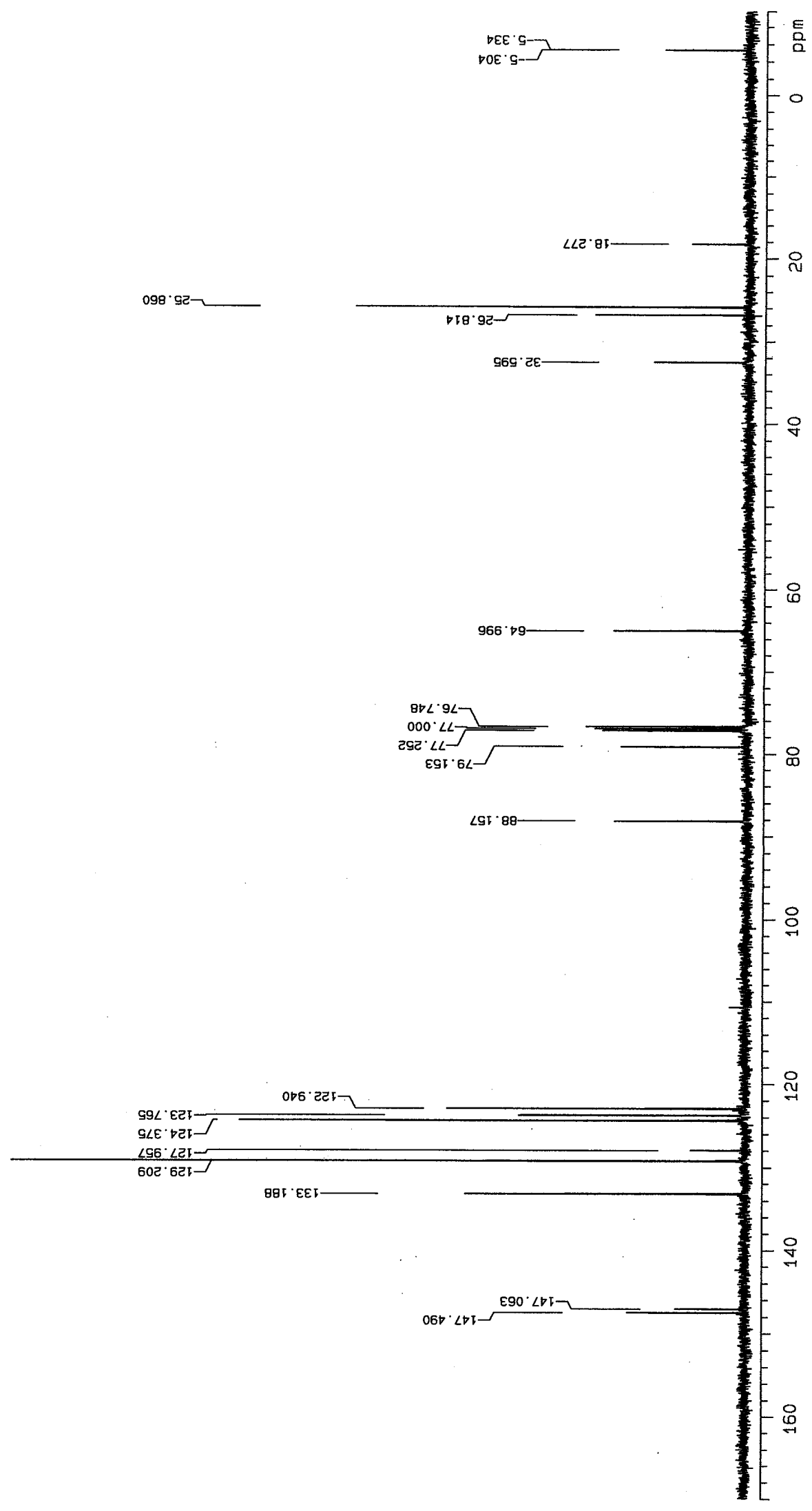




$$
\text { TBDMSO ح- - }
$$

${ }^{1} \mathrm{H}$ NMR of $\mathbf{1 c} \beta$

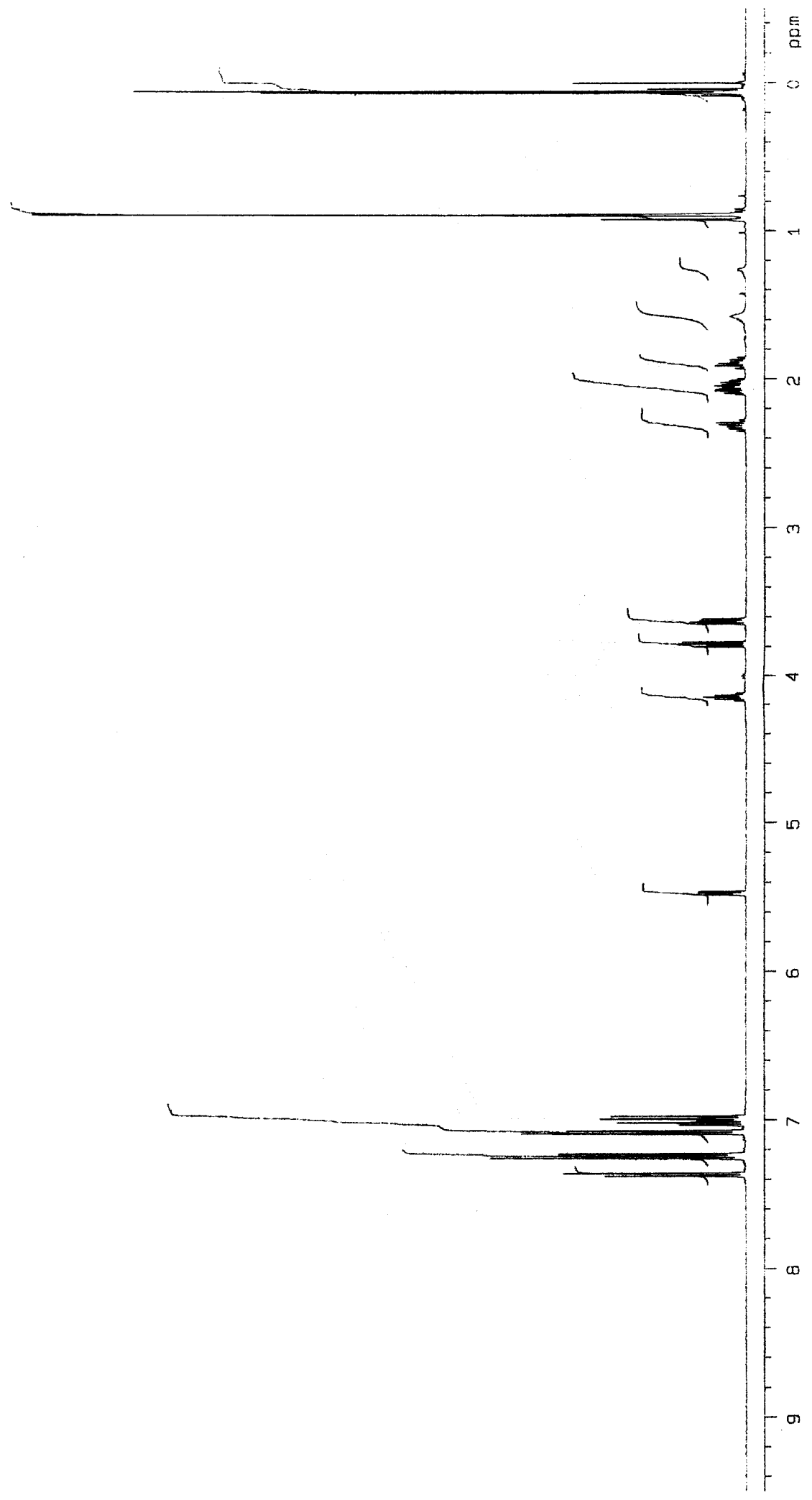


${ }^{13} \mathrm{C}$ NMR of $\mathbf{1 c} \beta$

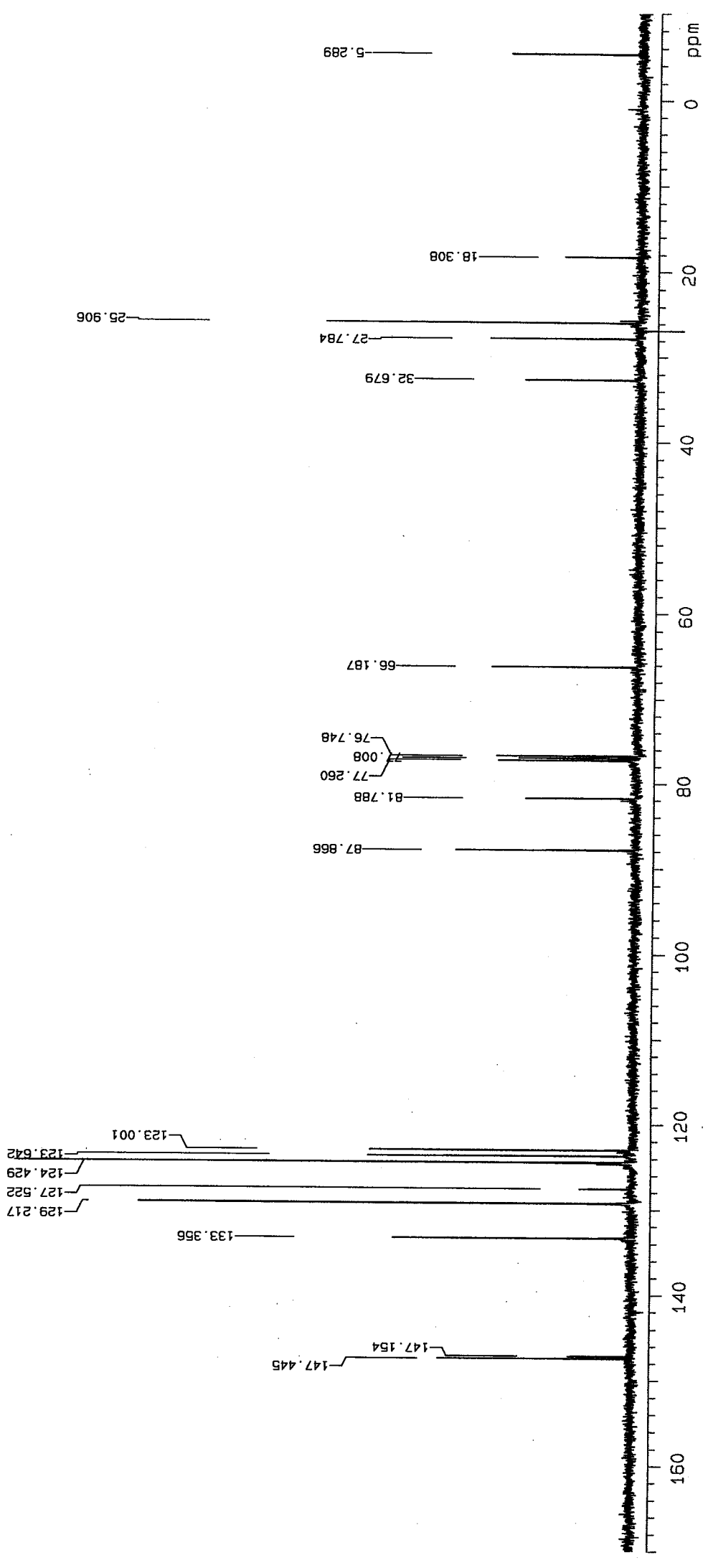




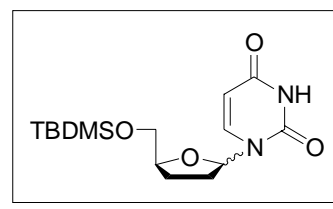

${ }^{1} \mathrm{H}$ NMR of dideoxynucleoside 3

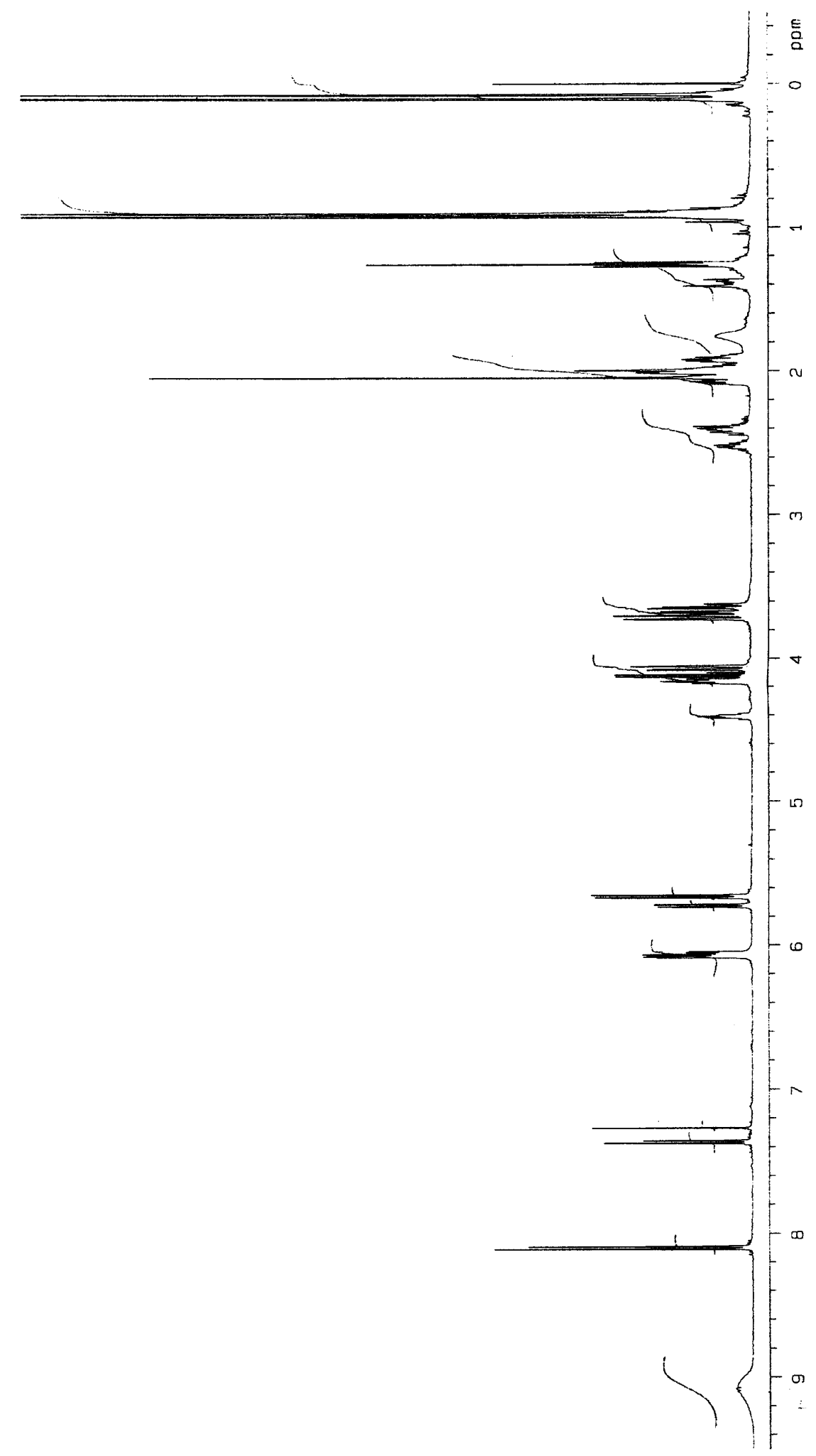




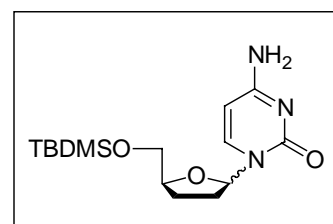

${ }^{1} \mathrm{H}$ NMR of dideoxynucleoside 4

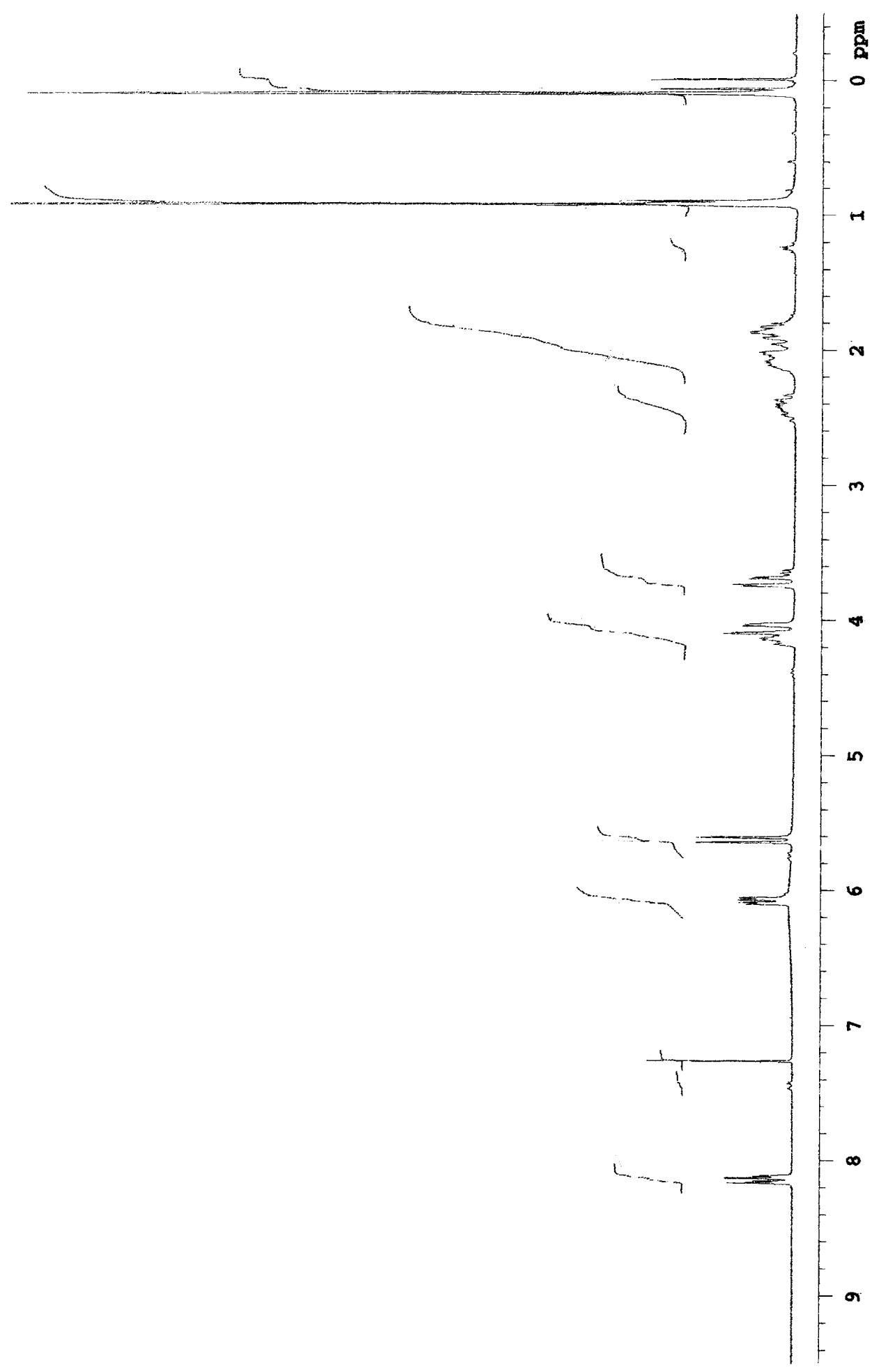




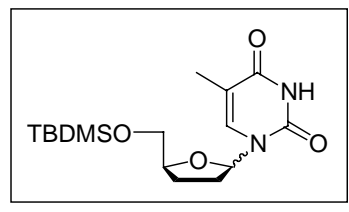

${ }^{1} \mathrm{H}$ NMR of dideoxynucleoside 5

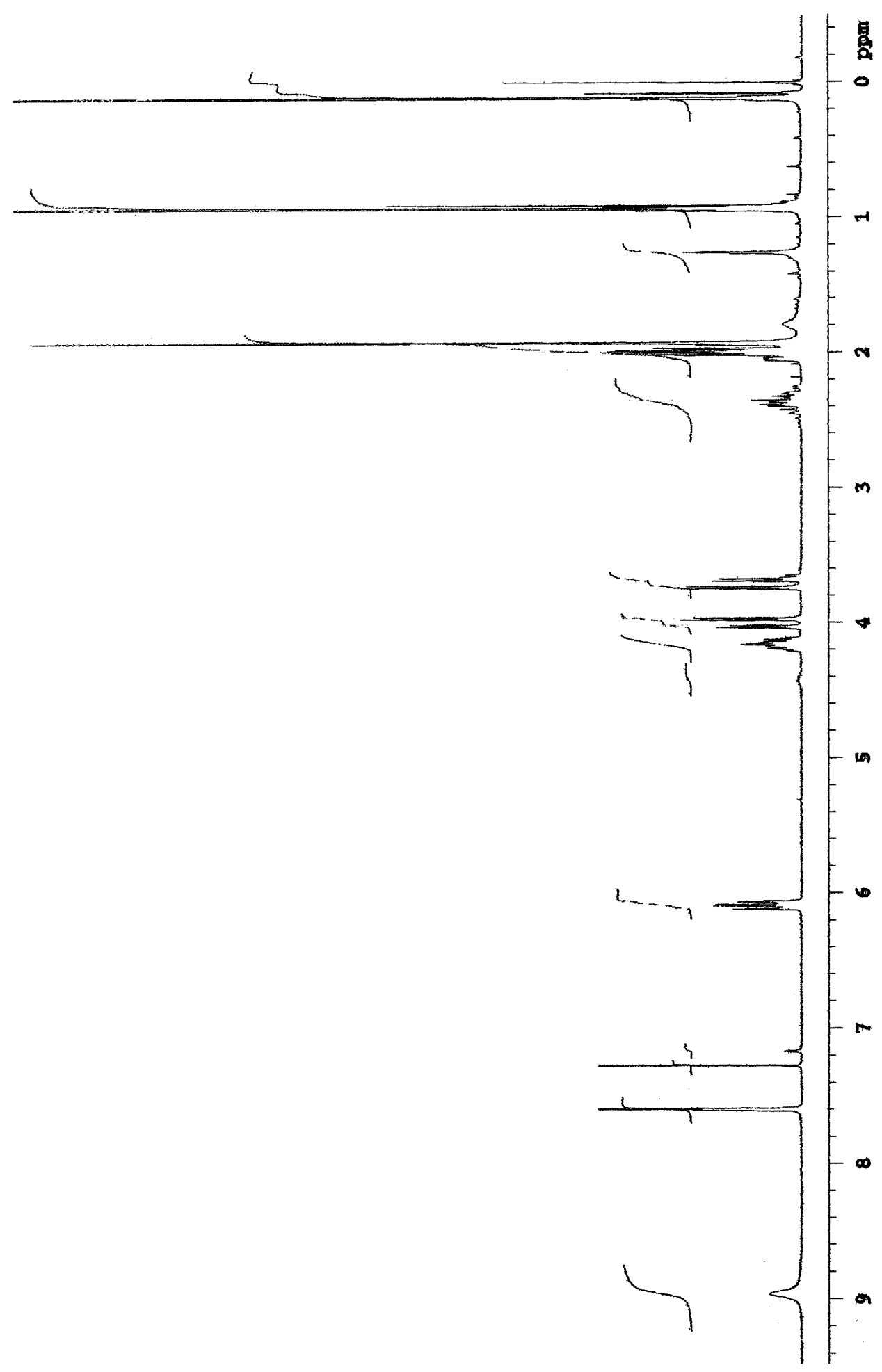




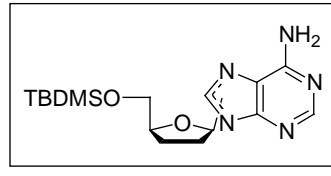

${ }^{1}$ H NMR of dideoxynucleoside 6

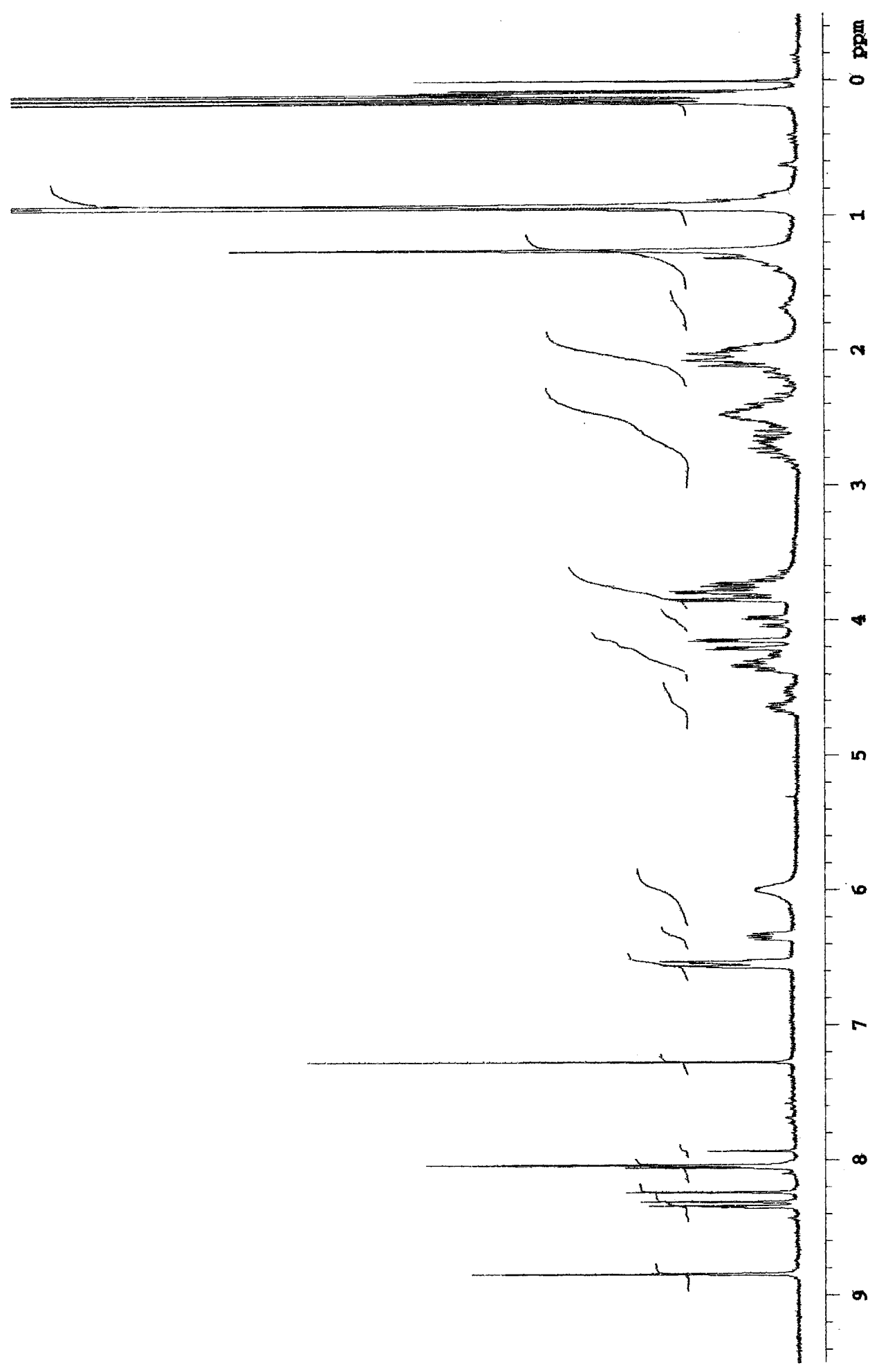


${ }^{1} \mathrm{H}$ NMR of the intermediates of the electrooxidation of $\mathbf{1 a} \alpha$ in $\mathrm{CD}_{2} \mathrm{Cl}_{2}$ at $-50{ }^{\circ} \mathrm{C}$

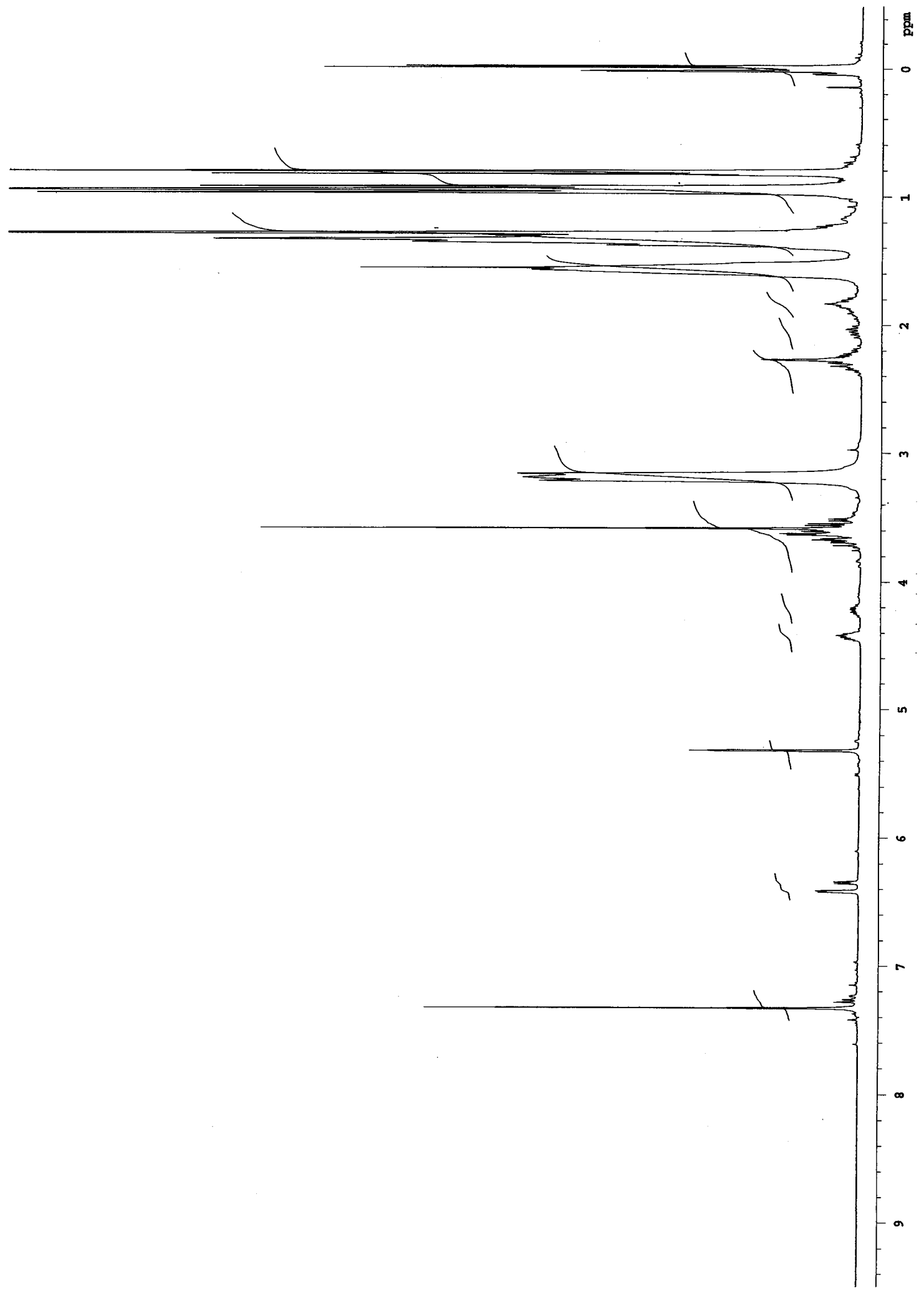

